\title{
Experimental and numerical investigation on the performance of hydrodynamic cavitation in multi-holes orifice plate
}

\author{
Changhao $\mathrm{Ji}^{1, \mathrm{a}}$, Zhixia $\mathrm{He}^{1,2, \mathrm{~b}^{*}}$, Yuhang Chen ${ }^{1, \mathrm{c}}$ \\ ${ }^{1}$ School of Energy and Power Engineering, Jiangsu University, Zhenjiang,Jiangsu 212013 , \\ ${ }^{2}$ Institute for Energy Research, Jiangsu University, Zhenjiang, Jiangsu 212013, China \\ a1203936875@qq.com, bx zxe@ujs.edu.cn, ${ }^{\text {c5 } 564339221 @ q q . c o m}$
}

\begin{abstract}
Key words: multi-holes orifice plate; cavitation; visualization; numerical simulation
Abstract: The multi-holes orifice plate are simple hydrodynamic devices which can be used for intensification of liquid-liquid heterogeneous micro-mixture for preparing bio-diesel or emulsified diesel. In this study,a flow visualization experiment system with a transparent hydrodynamic cavitation reactor was setup to investigate the cavitation in the orifice plate and the outlet of the orifice plate. The effect of upstream pressure and cavitation number were investigated. The experimental results show that with the increasing of upstream pressure the caviation occurs in the orifice plate and the outlet of the orifice plate which are correspond with the numerical results. The numerical results also show that cavitation cloud shedding are the source of the cavitation of the outlet of the orifice plate.
\end{abstract}

\section{Introduction}

Cavitation is a complex hydrodynamic phenomenon existed in liquid which can produce instantaneous high temperature and pressure. In addition, strong shock waves and micro-jets formed when it collapses in liquid [1]. From these properties, cavitation has attracted widely attention and has a broad application such as preparation of bio-diesel. There are many experimental and numerical researches on orifice plate structures such as opening ratio, thickness of orifice plate and shape of orifice at home and abroad [2-4]. Yu [5] studied the resistance characteristics of multi-holes orifice plate in hydraulic cavitation and used high-speed video camera to observe downstream of the orifice plate. However, few studies have been reported on cavitation in the orifice plate and downstream of the orifice export.

Hydrodynamic cavitation occurs due to orifice pressure declining to saturated vapor pressure of the liquid after high-speed liquid through multi-holes orifice plate and collapses at the end of the orifice and the downstream of pressure recovery area. Meantime, micro-jet and intense pressure fluctuation are generated which have a great effect on liquid-liquid heterogeneous mixture. Thus, it is important to research hydrodynamic characteristics of porous plate and cavitation characteristics in order to better design cavitation reactor for high energy efficiency.

\section{Experimental setup}

A visualized hydrodynamic cavitation experiment setup designed by ourselves was used to conduct the research and the schematic diagram of the experiment setup was shown in Fig. 1 which included multi-holes orifice plate cavitation reactor, circulation line system, image acquisition system. Cavitation reactor and orifice plate were made of transparent acrylic resin. Schematic diagram of shooting area of cavitation reactor was shown in Fig. 2. In the experiments, multistage centrifugal pump as a power cycle device was used to regulate the pressure in front of orifice plate and flow rate through reflux valve and bypass valve.

A high-speed CCD camera (MotionPro-TM10000, image size of $512 \times 512$ pixels at $10,000 \mathrm{fps}$ ) connected with a long distance microscope (Questar QM-1) was used to capture the Cavitation phenomena in the orifice plate. A high power LED light (99 LED bulbs) was installed at the 
opposite side of the camera across the orifice plate. Schematic diagram of shooting area of cavitation reactor was shown in Fig. 2. Mainly operating parameters of fluid medium and orifice plate used in this experiment are shown in Table 1.

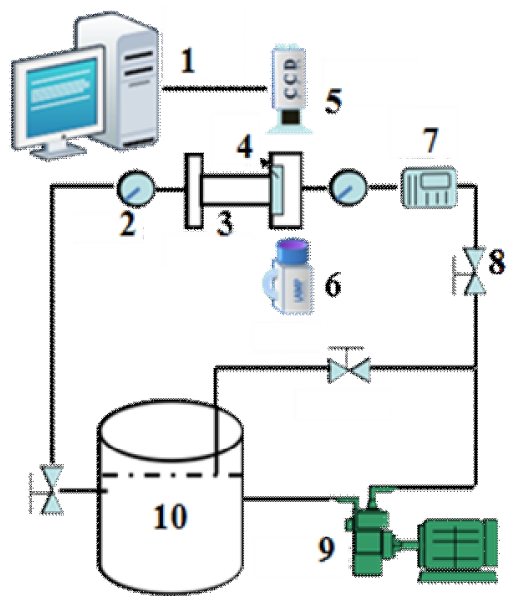

Fig.1 Schematic diagram of the tested experiment apparatus
1. acquisition system

2.Pressure gauge

3.Cavitation reactor

4.Orifice plate

5.High speed camera

6.LED light

7.Flow meter

8. Control valve

Table 1 The main operating parameters of fluid medium and orifice plate .

\begin{tabular}{lccc}
\hline contents & Parameters & contents & Parameters \\
\hline Fluid medium & $0^{\#}$ diesel & Orifice diameter $(\mathrm{mm})$ & 2 \\
Temperature $\left({ }^{\circ} \mathrm{C}\right)$ & $30^{\circ} \mathrm{C}$ & Orifice opening rate & 0.032 \\
Fluid viscosity $(\mathrm{mpa}-\mathrm{s})$ & 3.1 & Orifice opening number & 5 \\
Fluid density $\left(\mathrm{g} / \mathrm{m}^{3}\right)$ & 830 & Orifice plate thickness $(\mathrm{mm})$ & 5 \\
\hline
\end{tabular}

\section{Numerical models}

In this study, the flow characteristics inside an orifice plate and downstream of an orifice plate were analyzed by using visualized experiment principally and numerical simulation secondarily. Large commercial CFD software ANSYS Fluent 15.0 was used to analyze flow field in a cavitation reactor. Due to relatively complex flow field in multi-holes orifice plates, a turbulence model that could simulate jet shear and vortex accurately was needed. Thus, the shear stress model(SST) k-w model [6-7] was proposed to simulate cativation behavior inside an orifice plate because it could not only have a better prediction effect in near wall region but also apply for flow region of high Reynolds number. The detailed equations can be defined as:

$\frac{\partial}{\partial t}(\rho k)+\frac{\partial}{\partial x_{i}}\left(\rho k u_{i}\right)=\frac{\partial}{\partial x_{j}}\left(\Gamma_{k} \frac{\partial k}{\partial x_{j}}\right)+G_{k}-Y_{k}+S_{k}$

$\frac{\partial}{\partial t}(\rho \omega)+\frac{\partial}{\partial x_{i}}\left(\rho \omega u_{i}\right)=\frac{\partial}{\partial x_{j}}\left(\Gamma_{k} \frac{\partial \omega}{\partial x_{j}}\right)+G_{\omega}-Y_{\omega}+D_{\omega}+S_{\omega}$

where $\mathrm{k}$ is the turbulent kinetic energy. $\omega$ is turbulence frequency. $\rho$ is density of mixture. $\Gamma_{k}$ and $\Gamma_{\omega}$ are the diffusion rate of $\mathrm{k}$ and $\omega$, respectively. $Y_{k}$ and $G_{\omega}$ are the turbulence caused by turbulence, respectively.

The computational mesh that used in this work consisted of approximately 1.2 million purely hexahedral cells, refined holes and downstream of the orifice plate as shown in Fig. 3. The upstream and downstream of the orifice plate were 15 and $75 \mathrm{~mm}$,respectively. Considering boundary conditions in this work, pressure-inlet was at the range of $0.1 \sim 0.36 \mathrm{Mpa}$ and pressure outlet was kept constant corresponding with ambient pressure. 


\section{Results and discussion}

\section{Effect of upstream pressure on discharge coefficient}

Relationships of cavitation number $\mathrm{C}_{v}$ and discharge coefficient $\mathrm{C}_{\mathrm{d}}$ with different upstream pressure $p_{i}$ are given in Fig. 4. It could be seen that cavitation number $C_{v}$ decreased with the increased of upstream pressure $\mathrm{p}_{\mathrm{i}}$. In the case of upstream pressure up to $0.19 \mathrm{Mpa}$, incipient cavitation was occurred and cavitation number $\mathrm{C}_{v}$ was up to 1.12. As the inlet pressure continued to increase, cavitation developed and then extended to the end of the orifice plate,namely super cavitation. After that cavitation number $\mathrm{C}_{v}$ declined smoothly. In theory, the smaller the cavitation number, the more better the effect of cavitation would be. Incipient cavitation was occurred in the presence of $\mathrm{C}_{v}$ slightly larger than 1 because of tiny impurities contained in the fluid which caused the increase of cavitation nuclei. Cavitation nuclei were an essential factor for generating cavitation.

Discharge coefficient kept near 0.66 at the period of single flow and then it markedly increased with increasing pressure, while the maximum value of 0.673 was obtained at approximately 0.26 Mpa. Above $0.26 \mathrm{Mpa}$, the tendency went opposite. It could be concluded that attached cavitation were found near the walls of pipe and the resistance between fluid and pipe walls was greatly reduced. Thus, discharge coefficient had an obvious rise during the process of cavitation development. With the cavitation developed to super cavitation flow, cavitation area grew rapidly and large amount of cavitation bubbles got together under the downstream of the orifice plate. Instead of complete collapse, the majority of these formed choking flow that led an increase of resistance. In this work, the optimum upstream pressure was at the range of $0.26 \mathrm{Mpa}$ to $0.3 \mathrm{Mpa}$, namely between cavitation development and super cavitation.

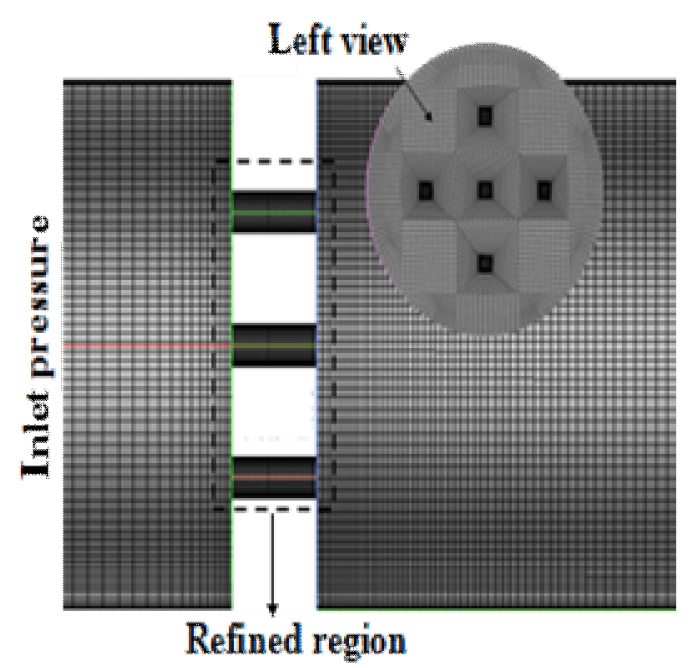

Fig. 3 Computational mesh of the reactor

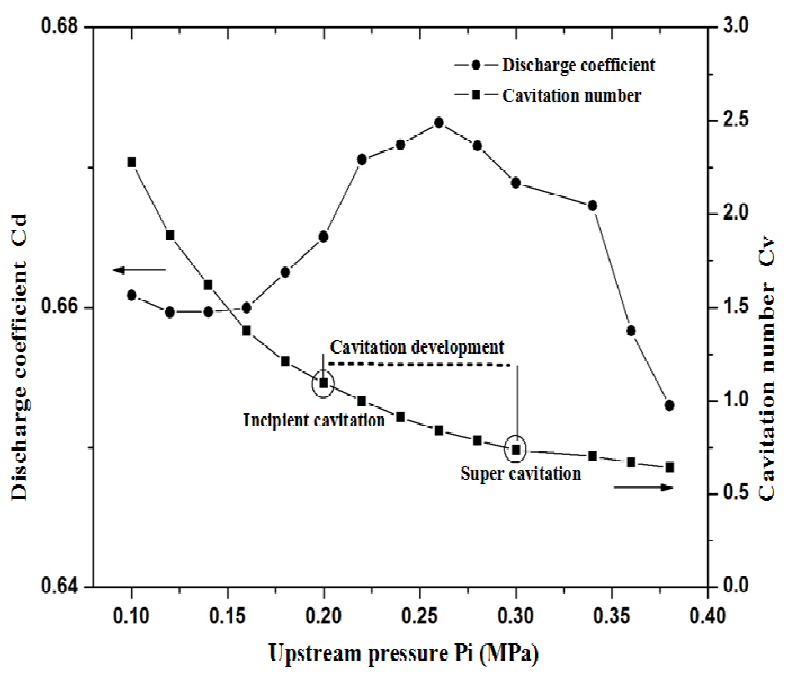

Fig. 4 Relationships of discharge coefficient and cavitation number at different upstream pressure

\section{Visualized experiment and numerical simulation}

Visualized experiment and numerical simulation were performed to make a detailed comparison as shown in Fig. 5. It could be founded that cavitation occurred at the corner of the orifice initially and then developed along with the axis of orifice formed the sheet cavitation eventually.

As cavitation number decreased due to increasing inlet pressure, development of cavitation became more and more intense and extended to export of the orifice plate, produced choking flow. However, cavitation region from numerical simulation was less than that from the experiment. On the one hand, errors came from the experiment and numerical simulation. On the other hand, as tiny bubbles were not totally collapsed but formed big bubbles which moved together with the high-speed liquid to the downstream of the orifice plate. In addition, high-speed CCD camera captured displacement superposition images of cavitation bubbles in a very short time. Therefore, 
compared the experiment and numerical simulation, cavitation region from experiment was higher relatively.

It is interesting to note that cavitation region was found under the downstream of the orifice plate from visualized experiment. This was different from numerical simulation which did not produce cavitation under the downstream of the orifice plate. It could be concluded that cavitation region produced under the downstream of the orifice plate was not due to pressure lower than saturated vapor pressure of the liquid but due to shedding of cloud cavitation and non-totally collapsed cavitation bubbles. In general, tiny cavitation bubbles formed big bubbles in the case of relatively low pressure of the vortexes near the wall and jets under the downstream. Thus, large amount of swirl bubbles was observed clearly under the downstream of the orifice plate in the experiment. The mechanical energy and chemical energy of cavitation collapse were applied by using hydrodynamic cavitation devices. In terms of non-totally collapsed cavitation bubbles, increasing back pressure slightly was taken into consideration for making these bubbles collapse totally.

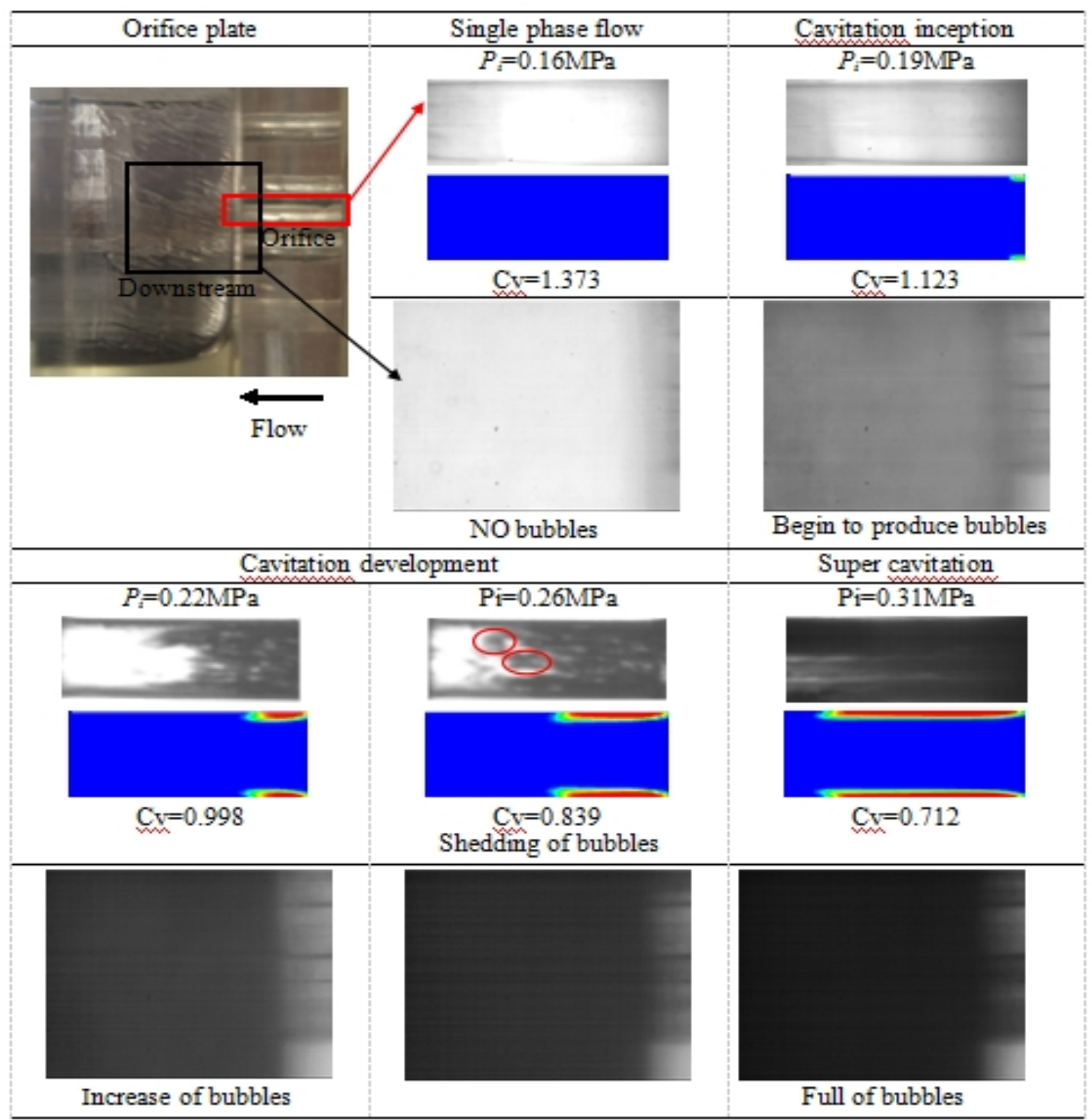

Fig. 5 Visualized experiment and numerical simulation

\section{Conclusions}

The hydraulic characteristics inside an orifice plate and downstream of an orifice plate were investigated based on visualized experiment and numerical simulation. The conclusions are listed as follows: 
As upstream pressure increased, cavitation number declined and then phase change occurred in presence of a low-pressure region, below the saturated vapor pressure of the liquid.Incipient cavitation number was 1.12 and super cavitation number was 0.712 .

Discharge coefficient had a marked rise when cavitation occurred and sharply decreased after the super cavitation.

SST constant simulation could predict the result of average flow field which corresponded with experimental results in a certain degree. And cavitation region under the downstream of the orifice plate was result of the shedding of cloud cavitation and non-totally collapsed cavitation bubbles.

\section{References}

[1] GOGATE P R. Cavitation: an auxiliary technique in wastewater treatment shemes [J]. Advances in Environmental Research, 2002, 6 ( 3) :335-358.

[2] V.K. Saharan, A.B. Pandit, P.S. Satish Kumar, S. Anandan, Hydrodynamic Cavitation as an Advanced Oxidation Technique for the Degradation of Acid Red 88 Dye, Ind. Eng. Chem. Res. 12 (2011) 1981-1989.

[3] Maynes D, Holt GJ, Blotter J. Cavitation inception and head loss due to liquid flow through perforated plates of varying thickness. ASME J Fluid Eng 2013;135:131302.

[4] P.N. Patil, P.R. Gogate, Degradation of methyl parathion using hydrodynamic cavitation: effect of operating parameters and intensification using additives, Sep. Purif. Technol. 95 (2012) 172-179.

[5] DongmeiYu, Xiaojiang Lu, jing Li, Research on Resistance Characteristics of Multi-hole Orifice Plate in Hydraulic Cavitation [J]. Light Industry Machinery. 2010, 28(1): 17-22, In chinese.

[6] Hongshi Yu, Tao Zhang, Shanshan Zhao, Wei Jiang. Simulation of the Flow Field of Multi-Hole Orifice Flow Meter [J]. Journal of Tianjin University, 2014, 47(1): 61-66, In chinese.

[7] Yanmei Li, Ying Xu, Liwei Zhang, et al. Simulation and experiment investigation on effect of upstream pipe single elbow on the performance characteristics of V-Cone flowmeters [J]. Chinese Journal of Scientific Instrument, 2009,30(6): 1195-1201, In chinese. 